\title{
Size quantization effects in InAs self-assembled quantum dots
}

\author{
K. H. Schmidt, ${ }^{\text {a) }}$ G. Medeiros-Ribeiro, ${ }^{\text {b) }}$ J. Garcia, and P. M. Petroff \\ QUEST and Materials Department, University of California, Santa Barbara, California 93106
}

(Received 13 November 1996; accepted for publication 25 January 1997)

\begin{abstract}
We study size quantization effects in InAs self-assembled quantum dots (QDs) that are embedded in GaAs. Using capacitance, photoluminescence and photovoltage spectroscopy, we correlate the measured quantized level energies with the quantum dot sizes and densities obtained from transmission electron microscopy. With increasing dot size, we observe a strong redshift of the QD features in all our data. In the capacitance spectra, a band gap renormalization of the two-dimensional wetting layer system appears when the first excited QD state crosses the wetting layer ground state. The relative size dependence and absolute energetic position of the QD transitions determined with photoluminescence provide some information about the influence of lateral confinement and height of the QD. (C) 1997 American Institute of Physics.

[S0003-6951(97)01913-X]
\end{abstract}

Zero-dimensional (0D) systems show not only interesting physical properties, they also promise new applications like single electron transistors, ${ }^{1}$ lasers, ${ }^{2}$ and memory devices ${ }^{3}$ with improved performance.

Strained layer epitaxy is one possible method to fabricate such OD quantum dots (QDs). The work described here focuses on the InAs/GaAs system. The 7\% lattice mismatch between the epitaxial film and the GaAs is used to induce the Stransky-Krastanow growth mode where the twodimensional (2D) growth characteristic changes into a threedimensional (3D) one after the deposition of about 1.5 monolayers (MLs) of InAs. ${ }^{4}$ Small InAs islands are formed on top of the thin InAs wetting layer (WL). The density and size of these islands depend on the amount of InAs deposited on the GaAs substrate. ${ }^{4}$ Although there are many publications about island growth and results of their spectroscopy, ${ }^{5-10}$ a careful study of size quantization effects as a function of InAs coverage is still lacking.

The wafer under investigation was fabricated by molecular beam epitaxy (MBE) deposition on a (100) GaAs semiinsulating substrate in a Varian Gen II system under an As pressure of $7 \times 10^{-5}$ Torr. At the initial stage of the growth 40 periods of a GaAs/AlAs $(2 \mathrm{~nm} / 2 \mathrm{~nm})$ short period smoothing superlattice was grown at a substrate temperature of $600{ }^{\circ} \mathrm{C}$. The 20-nm-thick Si-doped GaAs back contact $\left(n=10^{18} \mathrm{~cm}^{-3}\right.$ ) is separated from the smoothing superlattice by $200 \mathrm{~nm}$ of intrinsic GaAs. The spacer layer between the InAs QD system and the back contact consists of $30 \mathrm{~nm}$ undoped GaAs. The InAs WL layer and the QDs were grown at a pyrometer temperature reading of $530{ }^{\circ} \mathrm{C}$. To obtain QDs of varying size and density under identical growth conditions, wafer rotation was stopped only during the deposition of the InAs layer until the 2D-3D growth transition was observed in the reflection high-energy electron diffraction (RHEED) pattern. Due to the source geometry in the MBE machine it is possible to achieve a $0.2 \mathrm{ML}$ variation of InAs coverage across a 2 in. wafer. The InAs island system is

\footnotetext{
${ }^{\text {a)} P r e s e n t ~ a d d r e s s: ~ L e h r s t u h l ~ f u ̈ r ~ W e r k s t o f f e ~ d e r ~ E l e k t r o t e c h n i k, ~ R u h r-~}$ Universität Bochum, Universitätsstrasse 150/IC2, D-44780 Bochum, Germany. Electronic mail: schmidt@1we.ruhr-uni-bochum.de

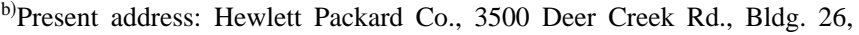
Palo Alto, CA 94304-1392.
}

capped by $5 \mathrm{~nm}$ of GaAs and the temperature is ramped to $600{ }^{\circ} \mathrm{C}$. The top of the structure consists of seven periods of a GaAs/AlAs (2 nm/2 nm) short period superlattice followed by $10 \mathrm{~nm}$ of GaAs to prevent sample oxidation. The Schottky contacts used for our capacitance and photovoltage experiments are made of $200 \mathrm{~nm}$ gold on top of $10 \mathrm{~nm}$ of $\mathrm{Ni}$ and $15 \mathrm{~nm}$ of $\mathrm{Cr}$. A Ni/AuGe alloy annealed at $400{ }^{\circ} \mathrm{C}$ for 120 s provided the ohmic back contact.

Plan view transmission electron micrographs (TEMs) were taken from a wafer position with low and high InAs coverage. In both images the InAs QDs are clearly observed through their associated strain field. ${ }^{11}$ The QD density in the samples under investigation changes from $\approx 2 \times 10^{9}$ to $\approx 5$ $\times 10^{10} \mathrm{~cm}^{-2}$. It is also important to note that the amount of InAs deposited is not high enough to cause dislocated island formation which should occur at a nominal InAs coverage larger than $\approx 2.5 \mathrm{ML}$ for our growth conditions. ${ }^{4}$ Hence, only the physics of coherently strained islands will be discussed. With the weak beam TEM imaging technique we determined the lateral dimensions of our QDs to be around $20 \mathrm{~nm}$. The lateral dimension changes only slightly with the InAs coverage. The QDs have an approximately square truncated pyramidal base shape with one set of facets parallel to (311) planes. ${ }^{12}$ With cross section TEM we measured a QD thickness of $\approx 3 \mathrm{~nm}$. Although we studied the QD thickness on only a few samples, we expect it to vary with the position on the wafer. This variation is inferred from previous atomic force microscopy measurements. ${ }^{4,13,14}$ However, since strain contrast is also present in the TEM images it is very difficult to get precise size information with the TEM technique.

The capacitance $(C-V)$ spectra shown in Fig. 1 are measured at five different wafer positions, i.e., InAs coverages shown in the lower-left corner of Fig. 1. Experimental details of our $C-V$ experiments are described elsewhere. ${ }^{15}$ At high InAs coverage all the dot levels are above the Fermi level for $U \leqslant 0.35 \mathrm{~V}$ (Fig. 1 spectrum 1). Here, the capacitance is only determined by the doping concentration in the backgate and the thickness of the total intrinsic region above. As shown previously, ${ }^{8,15,16}$ with increasing forward bias the electron ground state is shifted below the Fermi energy $E_{F}$ and two electrons tunnel into the QD. The tunneling event is de- 


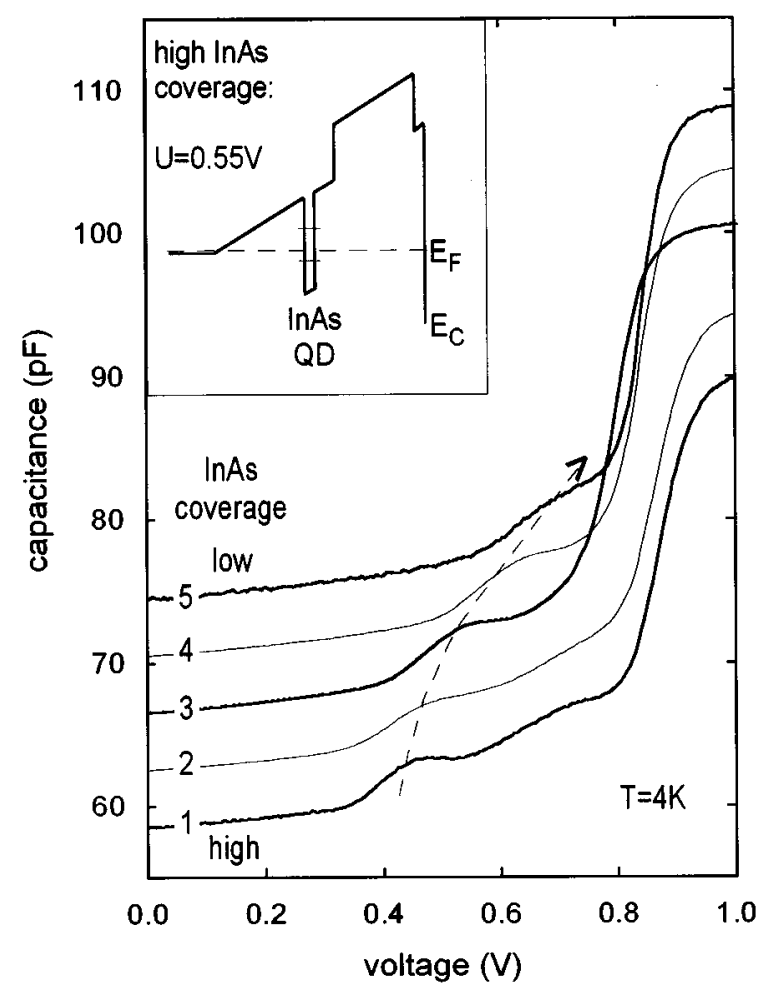

FIG. 1. Capacitance spectra measured at different wafer positions (i.e., InAs coverage or QD density and size). The voltage was modulated with $5 \mathrm{mV}$ and $500 \mathrm{~Hz}$. A constant offset is added to traces 2-5 for clarity. The dashed arrow indicates the position of the ground state of the QDs. The inset shows the conduction band structure at about $U=0.55 \mathrm{~V}$.

scribed by a peak between $0.35 \mathrm{~V} \leqslant U \leqslant 0.55 \mathrm{~V}$. The Gaussian line shape of the peak is caused by the size distribution of the InAs QDs. Due to the short lever arm Coulomb blockade effects cannot be observed in the sample investigated. ${ }^{8,15}$ An additional forward bias has to be applied to shift the first excited state below $E_{F}$ and to overcome the Coulomb blockade energy of those electrons that are already in the QDs. The corresponding spectral feature is the broad shoulder that appears between $0.55 \mathrm{~V} \leqslant U \leqslant 0.8 \mathrm{~V}$ when the first excited state is filled with the third, fourth, fifth, and sixth electron. Finally at $U \geqslant 0.85 \mathrm{~V}$ electrons tunnel from the back gate into the wetting layer which causes a dramatic increase in the capacitance signal. Only the ground and first excited QD states are observable in the $C-V$ spectrum of Fig. 1. Higher excited states are shifted into the WL continuum. $^{16}$

The thickness of the QDs decrease with InAs coverage. This effect was previously documented in Ref. 4. Thus the energy levels in the QDs are shifted to higher energies and tunneling into the ground state occurs at higher forward bias which is clearly observable in the $C-V$ spectra of Fig. 1 (dashed arrow).

Since a change in the InAs coverage mainly influences the QD and not the WL system, the $C-V$ traces $1,2,4,5$ remain almost constant above $U \geqslant 0.8 \mathrm{~V}$. However, in the $C-V$ trace 3 the onset of the WL feature appears at much lower forward bias compared to the other curves. We tentatively attribute this effect to a level crossing between the first excited QD state and the WL ground state. In fact in $C-V$

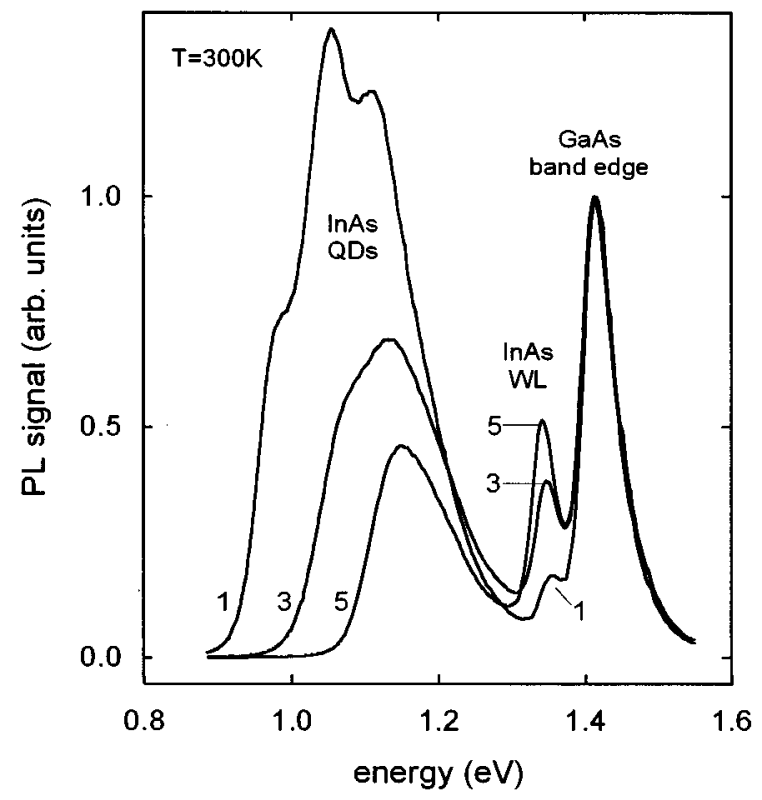

FIG. 2. Photoluminescence spectra measured at wafer positions 1,3 , and 5 (cf. Fig. 1) pumped with $\approx 10 \mathrm{~W} / \mathrm{mm}^{2}$ from a focused $\mathrm{Ar}^{+}$laser. The spectra are normalized to the GaAs band edge.

traces 1 and 2, tunneling into the first excited state is clearly observable while in $C-V$ traces 4 and 5 only the ground state feature appears. In the latter spectra the first excited QD state is already shifted into the WL continuum. When the first excited QD state crosses the WL ground state and forms a shallow bound state, its wave function extends over a large distance. Adjacent QDs couple with each other and form a quasi-two-dimensional electron gas which lowers the ground state of the $2 \mathrm{D}$ system.

Figure 2 displays photoluminescence (PL) spectra measured at high (curve 1), intermediate (curve 3), and low (curve 5) InAs coverage. The data were taken at the same wafer positions as those corresponding to the numbered $C-V$ curves of Fig. 1. All spectra are normalized to the GaAs band edge, which is clearly observable at $\approx 1.42 \mathrm{eV}$. Since the InAs wetting layer has a thickness of only $\approx 1.5$ ML, its ground state transition occurs right below the GaAs band edge at $1.34 \mathrm{eV}$. Since the height of the QD (thickness of the QD in growth direction $z$ ) is much larger than the thickness of the WL and the lateral confinement of the island is relatively weak compared to the one in the $z$ direction, the QD PL appears below the WL ground state between 0.9 and $1.3 \mathrm{eV}$. With increasing dot density more and more carriers relax from a two-dimensional WL into zero-dimensional QD levels which results in a decreasing PL signal of the WL while that of the QDs increases in intensity. There are three possible reasons for the slight blueshift of the WL transition at high InAs coverage. Due to the strain potential surrounding each dot a decrease in dot distance (at high QD density) might result in a higher strain in the WL system in between the dots. The strain field shifts the WL states to higher energies. Another possible reason is that material for island formation is also taken from the WL which would result in a decrease in average WL thickness or in an increase of $\mathrm{In} / \mathrm{Ga}$ intermixing. Both effects would also lead to the observed 
blueshift of the WL ground state transition observed in Fig. 2.

As expected, a change in InAs coverage, i.e., InAs QD size and density, mainly influences the QD PL itself. Along with a strong increase in intensity, the onset of the QD PL signal shows a redshift of about $150 \mathrm{meV}$ which reflects the increase in QD size (mostly thickness). However, the energetic distance between QD transitions remains roughly constant. This effect can be explained by the strong asymmetry between the height and the diameter of the QD. Due to the strong confinement in the growth direction, the absolute energetic position of the QD PL is determined by the height of the QD whereas the energetic splitting between the energy levels is influenced by their lateral dimensions. Since the dot height is much smaller than its diameter, a change in the overall thickness of the QD has a big influence on the absolute energetic position of the QD PL. The energetic distance between the QD PL peaks, however, is less affected. These observations are also in good agreement with theoretical calculations. ${ }^{5-7}$ With increasing InAs coverage the full line width at half-maximum of each QD transition determined by a multiple Gauss fit decreases from $\approx 80$ to $\approx 60 \mathrm{meV}$ which is also a hint that more and more QDs reach their size limit at high InAs coverage. An additional increase in InAs coverage produces dislocated islands.

The inset of Fig. 3 shows the band structure of our sample with illumination. If there is absorption of the incident light in the intrinsic region, the excited electron hole pairs are separated by the electric field induced by the Schottky contact. The holes stay in the QD since the spacer layer between the InAs QD region and the $\mathrm{AlGaAs}$ barrier is only $5 \mathrm{~nm}$. The photon induced electrons, however, tunnel out of the QD and partially screen the space charge region which results in a photovoltage signal between the back gate and the front contact.

The size quantization effects observed in the $C-V$ and PL data appear also in the absorption of our sample which is reflected in the photovoltage (PV) spectra of Fig. 3. In order to compare our results with the $C-V$ and PL data, the PV signal was measured on the same Schottky diodes. Since the front contact consists of a thick gold alloy layer, we illuminate our sample from the back side. Therefore all the light with energy above $\approx 1.4 \mathrm{eV}$ is absorbed by the GaAs semiinsulating substrate and the PV signal in this energy range vanishes. Fortunately, the substrate is transparent at energies smaller than the GaAs band gap and enough light reaches the field region of the Schottky diodes. As soon as there is absorption either in the QDs or from the WL, the intrinsic field in the active region separates the carriers which results in a PV signal. Like in the PL spectra of Fig. 1 increasing InAs coverage (i.e., QD size and density) results in a redshift and an increase of QD absorption between 0.9 and $1.25 \mathrm{eV}$ (Fig. 2 ). The strong peak at $\approx 1.35 \mathrm{eV}$ reflects absorption into the ground state of the InAs WL. The blueshift of the WL absorption edge (see also Fig. 2) with respect to the cut off energy (caused by GaAs substrate absorption) results in a decrease of the WL signal at $1.36 \mathrm{eV}$.

In conclusion we used TEM to characterize the size and density of InAs QDs embedded in GaAs bulk material. The influence of these two parameters on the electronic structure

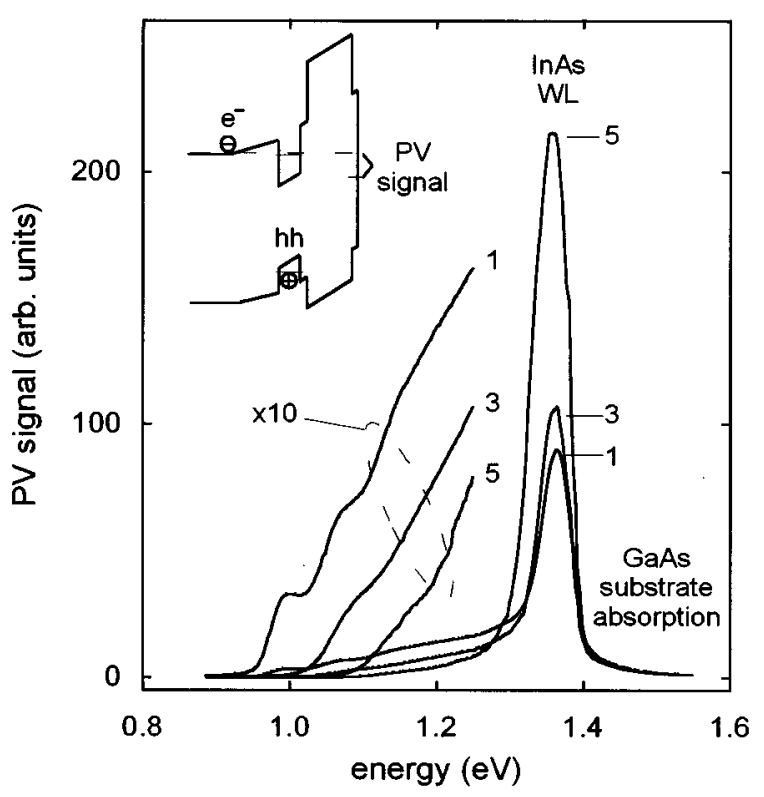

FIG. 3. Photovoltage spectra of samples 1,3 , and 5 taken at $T=300 \mathrm{~K}$ (cf. also Fig. 2). The sample was illuminated from the back side with the monochromatic light of a halogen lamp. The spectra are normalized to the signal above the GaAs absorption edge and multiplied by 10 for energies below $1.25 \mathrm{eV}$. The inset shows the band structure of the sample under illumination.

of our QDs was investigated by $C-V$, PL, and PV spectroscopies. In all our spectra a redshift of the QD features with increasing dot size is clearly observable.

The authors wish to acknowledge useful discussions with D. Leonard and M. Oestreich. This work was supported by QUEST, a NSF science and technology center (Grant No. DMR91-20007), the Alexander von Humboldt Foundation (KHS), and the Brazilian agency CNPq (GMR).

${ }^{1}$ N. Yokoyama, S. Muto, K. Imamura, M. Takatsu, T. Mori, Y. Sugiyama, Y. Sakuma, H. Nakao, and T. Adachihara, Solid-State Electron. 40, 505 (1996).

${ }^{2}$ H. Shoji, K. Mukai, N. Ohtsuka, M. Sugawara, T. Uchida, and H. Ishikawa, IEEE Photonics Technol. Lett. 7, 1385 (1995).

${ }^{3}$ G. Yusa and H. Sakaki, Electron. Lett. 32, 491 (1996).

${ }^{4}$ D. Leonard, K. Pond, and P. M. Petroff, Phys. Rev. B 50, 11687 (1994).

${ }^{5}$ A. Wojs, P. Hawrylak, S. Fafard, and L. Jacak, Phys. Rev. B 54, 5604 (1996).

${ }^{6}$ M. Grundmann, O. Stier, and D. Bimberg, Phys. Rev. B 52, 11969 (1995).

${ }^{7}$ J.-Y. Marzin, J. M. Gerard, A. Izrael, D. Barrier, and G. Bastard, Phys. Rev. Lett. 73, 716 (1994).

${ }^{8}$ H. Drexler, D. Leonard, W. Hansen, J. P. Kotthaus, and P. M. Petroff, Phys. Rev. Lett. 73, 2252 (1994).

${ }^{9}$ Y. Nabetani, T. Ishikawa, S. Noda, and A. Sasaki, J. Appl. Phys. 76, 347 (1994).

${ }^{10}$ S. Fafard, R. Leon, D. Leonard, J. L. Merz, and P. M. Petroff, Superlattices Microstruct. 16, 303 (1994).

${ }^{11}$ T. Benabbas, P. Francois, Y. Androussi, and A. Lefebvre, J. Appl. Phys. 80, 2763 (1996).

${ }^{12}$ J. Feng and P. M. Petroff (unpublished).

${ }^{13}$ J. M. Moison, F. Houzay, F. Barthe, L. Leprince, E. André, and O. Vatel, Appl. Phys. Lett. 64, 196 (1994).

${ }^{14}$ N. P. Kobayashi, T. R. Ramachandran, P. Chen, and A. Madhukar, Appl. Phys. Lett. 68, 3299 (1996).

${ }^{15}$ G. Medeiros-Ribeiro, D. Leonard, and P. M. Petroff, Appl. Phys. Lett. 66, 1767 (1995).

${ }^{16}$ K. H. Schmidt, G. Medeiros-Ribeiro, M. Oestreich, G. H. Döhler, and P. M. Petroff, Phys. Rev. B 54, 11346 (1996). 